\title{
Transplant renal artery stenosis secondary to mechanical compression from polycystic kidney disease: A case report
}

\author{
Linda Lee, MD, ${ }^{*}$ Lakshman Gunaratnam, MD, MSc, ${ }^{\dagger}$ Alp Sener, MD, PhD ${ }^{*}$ \\ *Division of Urology, Department of Surgery, Western University, London, ON; 'Division of Nephrology, Department of Medicine, Western University, London, ON
}

Cite as: Can Urol Assoc J 2013;7:E251-3. http://dx.doi.org/10.5489/cuai.548

\section{Abstract}

Transplant renal artery stenosis (TRAS) is a potentially treatable cause of allograft dysfunction, hypertension and graft loss. The mainstay of treatment includes angioplasty and endovascular stenting, although observation and surgery are at times indicated. We present an unusual case of TRAS secondary to mechanical compression from a patient's enlarged native polycystic kidneys. This was treated with bilateral native nephrectomy and evidence of TRAS improved both clinically and radiographically. Recognition and treatment are important in preventing irreversible complications of TRAS.

\section{Introduction}

The incidence of transplant renal artery stenosis (TRAS) ranges from $1 \%$ to $23 \%$ and is a potentially reversible cause of allograft dysfunction, hypertension and graft loss. ${ }^{1}$ The incidence may be rising due increasing use of Doppler ultrasonography (US) and magnetic resonance angiography (MRA) for diagnosis. ${ }^{2}$ Causes, such as atherosclerosis, immune-mediated endothelial damage and anatomic factors (e.g., length discrepancy between the transplanted artery and vein), have been implicated. ${ }^{3}$ To date, treatment options include observation, transluminal angioplasty, metallic stents, drug-eluting stents and anastomotic revision. ${ }^{2}$

We present a case of TRAS secondary to mechanical compression of the transplant renal artery by polycystic native kidneys. Resolution of renal artery stenosis was seen clinically and radiographically after bilateral nephrectomy of the native kidneys, thus describing the first such case in the literature.

\section{Case report}

A 76-year-old male with end-stage renal failure secondary to adult polycystic kidney disease underwent cadaveric Epstein-Barr virus (EBV)-mismatched donation after cardiac death (DCD) kidney transplantation in the right lower quadrant in October 2009, after having been on hemodialysis for 1 year. With human leukocyte antigen (HLA) matching, he had single antigen mismatches at the HLA-A, HLA-B and HLA-DQ loci, two antigen mismatches on the HLA-DR and DRW loci and no mismatches on the HLA-C locus. Pre-transplant panel reactive antibody (PRA) was $0 \%$ for both Class I and Class II major histocompatibility complex (MHC) using a Luminex (Life Technologies, Inc., Burlington, $\mathrm{ON})$. The induction regimen consisted of thymoglobulin, prednisone, tacrolimus and mycophenolate mofetil (MMF). The patient had a normal course in hospital following transplantation and was discharged with a serum creatinine of $112 \mathrm{umol} / \mathrm{L}$. Three months following transplantation, the patient developed hypertension $(159 / 66 \mathrm{mmHg})$ which was refractory to multiple anti-hypertensive medications, which included labetalol and nifedipine. When he returned for his 8 month follow-up, his blood pressure was $189 / 85 \mathrm{mmHg}$ in the outpatient clinic and irbesartan was added. This was his last clinic appointment before he was admitted to hospital.

In July 2010, he presented with a 1-month history of fatigue, malaise, weakness and failure to thrive. On physical examination, it was found that he had an enlarged left-sided neck mass and a distended abdomen due to massive polycystic kidneys, which both felt to be of the same size as compared to the time of transplant. Although cytomegalovirus (CMV) serology was negative, EBV serology was positive at the time of presentation. He was admitted to hospital for further investigation and management. Biopsy of the neck mass was found to be positive for post-transplantation lymphoproliferative disorder (PTLD) and he was treated with a combination of a reduction in his immunosuppressive regi- 
men and the anti-CD20 monoclonal antibody, rituximab, by hematology. During his course in hospital, he continued to have refractory hypertension, with a systolic blood pressure $>200 \mathrm{mmHg}$, elevated creatinine of $187 \mathrm{umol} / \mathrm{L}$ and shortness of breath. Doppler US revealed evidence of renal artery stenosis of the transplanted kidney with a main renal artery resistive index of 0.80 and peak systolic velocity rates of $470.4,48.7,16.8$ and $14.7 \mathrm{~cm} / \mathrm{s}$ in the main, intralobar, upper and lower arcuate renal arteries, respectively, along with a distinctive parvus et tardus waveform. The stenotic region was at the anastomosis. Computed tomography (CT) angiography showed narrowing of the transplant renal artery, suggestive of renal artery stenosis. Dyspnea was investigated and there was no radiographic evidence of pulmonary edema, effusion or embolism. The shortness of breath was attributed to abdominal distension and decreased functional residual capacity due to the enlarged native kidneys (right kidney $25.0 \times 15.0 \times 15.0 \mathrm{~cm}$ and left kidney $30.0 \times 18.0 \times 14.0 \mathrm{~cm}$ ). It was thought that both the dyspnea and TRAS were related to mechanical compression, given the massive size of the native kidneys. The CT scan also confirmed our physical exam findings that the kidneys had not changed in size compared to pre-transplant measurements. After a discussion with the patient, he elected to undergo bilateral native nephrectomy for his massive polycystic kidneys (Fig. 1) and renal artery stenosis rather than endovascular stenting. Notwithstanding, if bilateral native nephrectomy failed to relieve the TRAS, an endovascular approach would remain an option.

Preoperatively, the patient's hypertension was poorly controlled on doxazosin, hydrochlorothiazide, irbesartan, labetalol, nifedipine and hydralazine. A bilateral nephrectomy was performed for symptom relief and treatment of
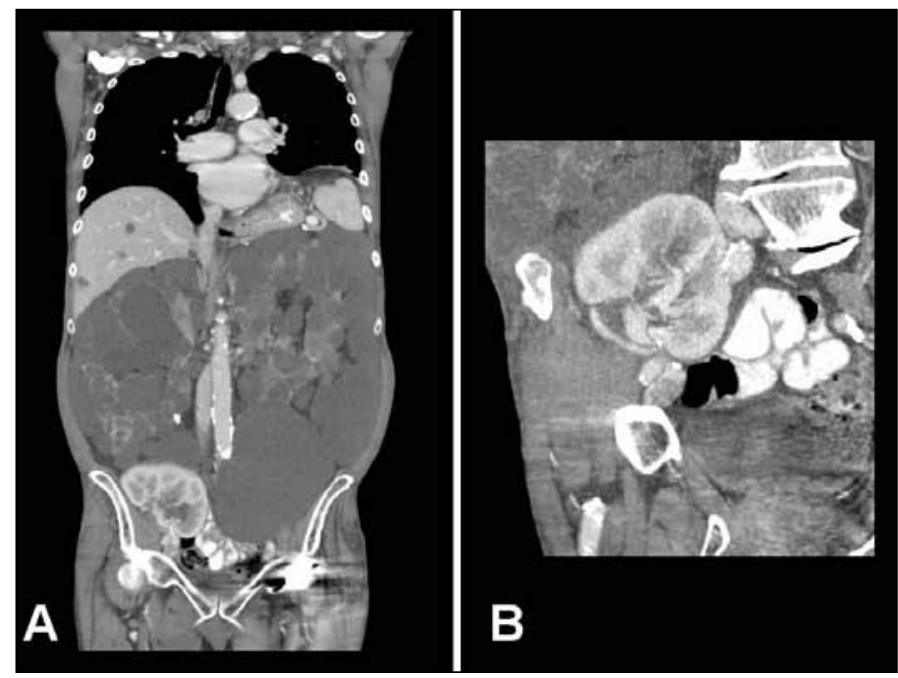

Fig. 1. Coronal view of the computed tomography scan of the abdomen and pelvis, which demonstrates massive polycystic kidney disease and transplanted kidney in the right iliac fossa. renal artery stenosis. During the operation, the transplanted renal artery was examined intraoperatively and no abnormalities were seen. Blood loss was minimal. The right and left kidneys weighed $3.2 \mathrm{~kg}$ and $4.4 \mathrm{~kg}$, respectively (Fig. 2).

Immediately following native nephrectomy, serum creatinine levels began to decline. Upon discharge on postoperative day 15 , he was normotensive on only nifedipine and labetalol and his creatinine had returned towards baseline values. Doppler US was repeated 1 month postoperatively, which showed a resolution of TRAS. The main renal artery resistive index was 0.56 and there was a return of normal renal artery waveform.

\section{Discussion}

The diagnosis of TRAS was made based on colour Doppler US in this patient. While angiography is considered to be the gold standard, Doppler US offers a non-invasive method of imaging without the risk of contrast nephropathy. A peak systolic velocity of $\geq 250 \mathrm{~cm} / \mathrm{sec}$ is associated with a sensitivity of up to $100 \%$ and a specificity of $95 \%$ for renal artery stenosis. ${ }^{4}$ The parvus-tardus waveform has also been associated with sensitivities as high as $95 \%,{ }^{5}$ although this characteristic alone may not be adequate for diagnosis. ${ }^{6}$ Additionally, a resistive index of $\geq 0.80$ at least 3 months after transplantation is associated with allograft failure and death with a functioning graft.?

TRAS typically arises at or close to the anastomotic site and is often related to vascular intimal hyperplasia or, if presenting years after transplant, progressive atherosclerosis. ${ }^{8}$ In this patient, TRAS documented on CT and colour Doppler US was markedly improved after bilateral native nephrectomy, which suggests a reversible etiology. In contrast to

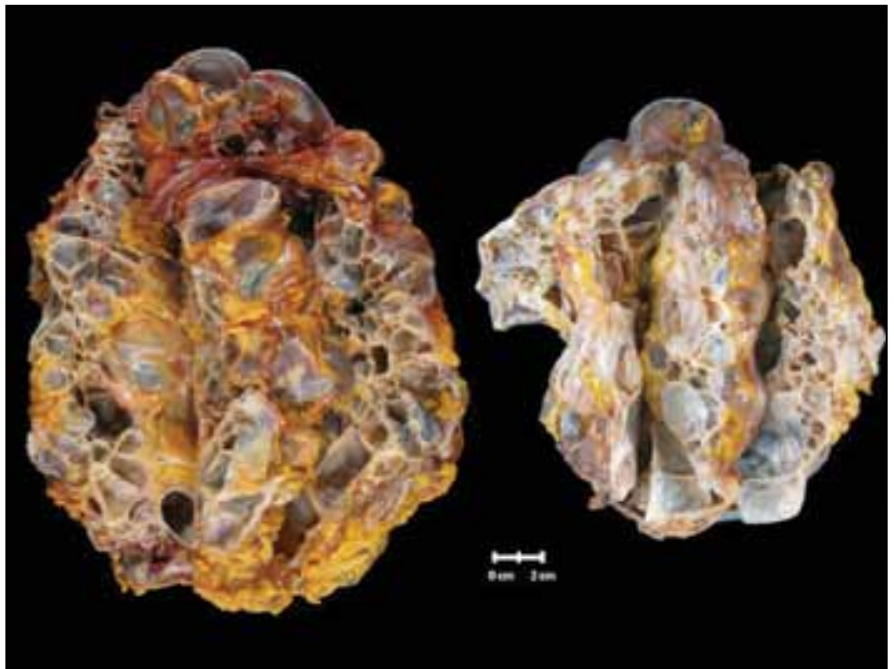

Fig. 2. Left and right kidneys demonstrating end-stage polycystic kidney disease. 
the typical presentation of TRAS from intimal hyperplasia or atherosclerosis, this patient may have developed TRAS from mechanical compression or kinking of the transplant renal artery. In addition, the enlarged polycystic kidneys may have also caused compression of the transplant kidney leading to a "Page kidney." This phenomenon was first described in an experiment by Irwin Page in 1939, where canine kidneys were wrapped in cellophane to induce arterial hypertension via activation of the renin-angiotensin-aldosterone pathway. ${ }^{9}$ A Page kidney, however, alone would not explain the radiographical findings on US.

Endovascular techniques, such as angioplasty and stenting, are the mainstay of TRAS management. Percutaneous transluminal angiography (PTA) and stent placement can improve renal function in $30 \%$ of cases and stabilize renal function in $38 \%$. In addition, hypertension can be cured or improved in $69 \%$ of patients. ${ }^{10}$ Studies of PTA with stenting have recurrence rates as low as less than $10 \% .{ }^{10}$ There is emerging evidence for drug-eluting stents to prevent restenosis. ${ }^{11}$ Surgery is also an option for failed angioplasty or severe stenoses not suitable for angioplasty and stenting. Surgery typically involves resection of the stenotic segment of the renal artery and revision of the anastomosis, but in rare cases, autotransplantation.

Given the mechanism of TRAS in this case and the location at the anastomosis, it is unlikely that the conventional techniques would have been sufficient. It is likely that the mechanical compression leading to TRAS was relieved following bilateral native nephrectomy. Following nephrectomy, clinical improvement was seen with regards to hypertension, renal function and dyspnea, as well as radiographic improvement in resistive indices and peak systolic flow.

\section{Conclusion}

This report describes the first case of TRAS secondary to mechanical compression of the patient's native kidneys, which was completely reversed with bilateral native nephrectomy. In select cases, it may be appropriate to perform bilateral native nephrectomy at the time of transplantation. Although we present a novel case in this report, it is important to be aware of this phenomenon as a potentially treatable cause of transplant renal artery stenosis.

Competing interests: None declared.

This paper has been peer-reviewed.

\section{References}

1. Fervenza $\mathrm{FC}$, Lafayette $\mathrm{RA}$, Alfrey $\mathrm{E}$, et al. Renal artery stenosis in kidney transplantation. Am I Kidney Dis 1998:31:142-8. http://dx.doi.org/10.1053/ajkd.1998.v31.pm9428466

2. Bruno S, Remuzzi G, Ruggenenti P. Transplant renal artery stenosis. J Am Soc Nephrol 2004:15:134-41. http://dx.doi.org/10.1097/01.ASN.0000099379.61001.F8

3. Patel NH, Jindal RM, Wilkin T, et al. Renal arterial stenosis in renal allografts: Retrospective study of predisposing factors and outcome after percutaneous transluminal angioplasty. Radiology 2001:219:663-7.

4. Baxter GM, Ireland H, Moss GJ, et al. Colour Dopper ultrasound in renal transplant artery stenosis: which Doppler index? Clin Radiol 1995:15:13.

5. Stavros AT, Parker SH, Yakes WF, et al. Segmental stenosis of the renal artery: pattern recognition of tardus and parvus abnormalities with Duplex sonography. Radiology 1992;184:487-92.

6. Kliewer MA, Tupler RH, Carroll BA, et al. Renal artery stenosis: analysis of Doppler waveform parameters and tardus-parvus pattern. Radiology 1993;189:779-87.

7. Radermacher J, Mengel $M$, Ellis $S$, et al. The renal arterial resistance index and renal allograft survival. $N$ Engl J Med 2003;349:115-24. http://dx.doi.org/10.1056/NEJMoa022602

8. Becker BN, Odorico JS, Becker YT, et al. Peripheral vascular disease and renal transplant artery stenosis: a reappraisal of transplant renovascular disease. Clin Transplant 1999;13:349-55. http://dx.doi. org/10.1034/i.1399-0012.1999.130412.x

9. Page IH. The production of persistent arterial hypertension by cellophane perinephritis. JAMA 1939;113:2046. http://dx.doi.org/10.1001/jama.1939.02800480032008

10. Leertouwer TC, Gussenhoven EJ, Bosch JL, et al. Stent placement for renal artery stenosis: Where do we stand? A meta-analysis. Radiology 2000;216:78-85.

11. Douis H, Shabir S, Lipkin G, et al. Drug-eluting stent insertion in the treatment of in-stent renal artery restenosis in three renal transplant recipients. J Vasc Interv Radiol 2008;19:1757-60. http://dx.doi. org/10.1016/i.jvir.2008.09.009

Correspondence: Dr. Alp Sener, Division of Urology, Department of Surgery, University Hospital, 339 Windermere Rd, London ON; fax: 519-663-3858; alp.sener@hsc.on.ca 